\title{
Développement d'une méthode primaire pour la détermination du fer total dans du sérum
}

\author{
Development of a primary method \\ for the determination of iron in serum
}

\author{
Marie PALOS ${ }^{1,2}$, Maria Estela DEL CASTILLO BUSTO ${ }^{1}$, Jean-Marc HATTCHOUEL ${ }^{3}$, Florence PANNIER ${ }^{2}$, \\ Paola FISICARO ${ }^{1}$ et Sophie VASLIN-REIMANN ${ }^{1}$ \\ 1 Laboratoire national de métrologie et d'essais (LNE), 1 rue Gaston Boissier, 75724 Paris Cedex 15, France, marie.palos@lne.fr, \\ Paola.Fisicaro@lne.fr. \\ 2 Laboratoire de Chimie Analytique Bio-Inorganique et Environnement (LCABIE), IPREM, Université de Pau et des Pays de l'Adour CNRS/UMR \\ 5254, Hélioparc, 2 avenue du Président Angot, 64053 Pau Cedex 09, France. \\ 3 Agence nationale de sécurité du médicament et des produits de santé (ANSM), Direction des dispositifs médicaux de diagnostic et des plateaux \\ techniques, 143/147 Bd Anatole France, 93285 Saint Denis Cedex, France.
}

\begin{abstract}
Résumé
Ce travail présente le développement d'une méthode de référence pour le dosage du fer dans du sérum par ICP-MS. Afin d'assurer la traçabilité métrologique des résultats, la dilution isotopique associée à l'ICP-MS (DI-ICP-MS) a été mise en place. De plus, cette méthode a l'intérêt d'être une méthode primaire. La validation de la méthode a été réalisée par l'utilisation d'un matériau de référence certifié (SRM 1598a) et la participation à une comparaison internationale entre laboratoires nationaux de métrologie (BIPM-CCQM K107). La méthode validée est maintenant utilisable par les laboratoires d'analyse biomédicale pour évaluer leurs capacités de mesure ou par ceux qui mènent des actions d'assurance qualité ou de contrôle qualité. En effet, cette méthode DI-ICP-MS peut être utilisée pour certifier des matériaux de référence ou pour attribuer des valeurs de référence aux échantillons utilisés dans le cadre d'essais d'aptitude. La faisabilité a été testée sur deux échantillons inconnus, le BCR ${ }^{\circledR}-637$ de l'IRMM et l'échantillon B17 de l'ANSM (matériau distribué au cours d'un programme du contrôle national de qualité des analyses de biologie médicale).
\end{abstract}

MOTS CLÉS : TRACABILITÉ MÉTROLOGIQUE, DILUTION ISOTOPIQUE, FER, SÉRUM, ICP-MS, MÉTHODE PRIMAIRE, ANALYSE BIOMÉDICALE.

\footnotetext{
Abstract

This work presents the development of a reference method by ICP-MS for the determination of iron in serum. To ensure the metrological traceability of the results, isotope dilution associated with ICP-MS
}

\begin{abstract}
(ID-ICP-MS) has been applied, which has the potential to be a primary measurement procedure. Method validation has been demonstrated through the use of a certified reference material (SRM 1598a) and the participation in an international comparison between National Metrology Institutes (BIPM-CCQM K107). The validated method is now available to support clinical laboratories in the evaluation of their measurement capabilities and for quality assurance and quality control programmes. In fact, this ID-ICP-MS method can be used to certify reference materials or to assign reference values to samples used in proficiency testing schemes. The feasibility has been tested on two unknown samples, i.e. BCR ${ }^{\circledR}-637$, IRMM sample and an ANSM B17 sample (a material distributed during a National Quality Control programme).
\end{abstract}

KEYWORDS: METROLOGICAL TRACEABILITY, ISOTOPE DILUTION, IRON, SERUM, ICP-MS, PRIMARY MEASUREMENT PROCEDURE, BIOMEDICAL ANALYSIS.

\section{Contexte de l'étude}

Le fer est un élément essentiel pour les organismes vivants qui l'utilisent pour la synthèse de protéines responsables du transport de l'oxygène (hémoglobine $\mathrm{Hb}$, myoglobine $\mathrm{Mb}$ ) ainsi que pour la formation des enzymes contenant du fer. Chez l'être humain, il existe principalement sous des formes complexes liées à des protéines. Ces ferroprotéines peuvent être classées en trois groupes : les composés contenant un noyau hème 
( $\mathrm{Hb}, \mathrm{Mb}$ ), les enzymes hémiques (cytochrome c peroxydase) et les composés non hémiques (ferritine Ft, transferrine Tf, enzymes flavines-fer) [1], avec une répartition type du fer de $60 \%$ provenant de l'Hb, $25 \%$ stockés essentiellement sous forme de ferritine et enfin $15 \%$ liés à $\mathrm{la} \mathrm{Mb}$ contenue dans les tissus musculaires [1]. Son transport dans le sérum se fait principalement après fixation sur la transferrine [2]. Le fer transporté sert à la synthèse de protéines mais aussi à l'approvisionnement des réserves en cet élément. Les principaux tissus de stockage cellulaire du fer sont le foie, la rate et la moelle osseuse. L'apport nutritionnel recommandé est de $8 \mathrm{mg}$ par jour pour un adulte (cette quantité est de $18 \mathrm{mg}$ par jour pour les femmes ménopausées). L'apport maximal toléré est de $45 \mathrm{mg}$ par jour [3]. Un adulte peut ainsi avoir entre $3 \mathrm{~g}$ et $4 \mathrm{~g}$ de fer dans son organisme [4]. Les valeurs physiologiques du fer dans l'organisme sont comprises entre $10 \mu \mathrm{mol} \cdot \mathrm{L}^{-1}$ et $30 \mu \mathrm{mol} \cdot \mathrm{L}^{-1}$ [5].

La conséquence la plus connue d'une carence en fer est l'anémie (teneur en fer inférieure à $5 \mu \mathrm{mol} \cdot \mathrm{L}^{-1}[6]$ ) qui est le résultat d'un stade avancé de carence. Cependant, d'autres maladies peuvent être rencontrées avant l'apparition de l'anémie, notamment, un état de fatigue physique et mentale due à une diminution du stockage de l'oxygène [7]. Dans le cas d'un excès de fer dans l'organisme, l'hémochromatose est la maladie la plus répandue (teneur en fer supérieure à $30 \mu \mathrm{mol} \cdot \mathrm{L}^{-1}$ [8]). C'est une maladie génétique due à un dysfonctionnement du stockage du fer qui entraîne son accumulation principalement dans le foie [9]. Sans traitement, cette maladie peut entraîner des atteintes hépatiques sévères (cirrhose, cancer hépatocellulaire), un dysfonctionnement sexuel... [10].

Le dosage du fer est largement réalisé par les laboratoires de biologie médicale : en 2014, 5,3 millions d'analyses pour le dépistage de l'anémie ont été effectués (7,3 millions d'euros remboursés) [11].

Compte-tenu de la gravité des pathologies associées aux teneurs anormales en fer et du nombre important d'analyses effectuées, la fiabilité des résultats de mesure pour le dosage du fer représente donc un enjeu majeur afin de disposer d'un diagnostic fiable et d'adapter au mieux les traitements. C'est dans cet objectif qu'en France, la réforme de la biologie médicale, adoptée en 2013, rend obligatoire l'accréditation par le Comité français d'accréditation (Cofrac) de tous les laboratoires de biologie médicale selon la norme NF EN ISO 15189 d'ici 2020 [12]. Cette norme implique que les laboratoires disposent d'un matériau ou d'une méthode de référence qui permettent de garantir la traçabilité des mesures au Système international d'unités (SI) [13]. Cependant, il n'existe ni méthode de référence, ni matériau de référence répertoriés sur le site du JCTLM (CIPM, IFCC and ILAC Joint Committee for Traceability in Laboratory Medecine pour le dosage du fer dans du sérum humain [14]. Ce comité a été créé en 2002 en réponse à la mise en œuvre de la Directive européenne 98/79/CE relative aux dispositifs médicaux de diagnostic in vitro. Il existe toutefois, à notre connaissance, deux matériaux de référence certifiés en fer dans du sérum. Le SRM 1598a Inorganic constituents in animal serum du NIST (laboratoire national de métrologie des États-Unis) est certifié par une méthode primaire (analyse par activation neutronique). Le JCCRM 322-5 Serum iron (sérum humain) est certifié par la méthode de référence définie par l'International Council for Standardization in Haematology (ICSH), qui n'est pas traçable aux unités SI. [15].

\section{Méthodes analytiques employées pour la mesure du fer dans le domaine biomédical}

A ce jour, il existe différentes méthodes de dosage du fer que l'on peut classer en trois groupes : les méthodes colorimétriques, les méthodes par spectrométrie d'absorption atomique et les méthodes par spectrométrie de masse [16]. Les techniques colorimétriques et par spectrométrie d'absorption atomique sont utilisées en routine dans les laboratoires de biologie médicale. Les méthodes utilisant la spectrométrie de masse ne sont quasiment utilisées qu'au sein de laboratoires de référence et de laboratoires nationaux de métrologie (LNM).

Les méthodes colorimétriques reposent sur l'ajout d'un chromogène à l'échantillon qui va se complexer avec le fer ferreux (Fe(II)) présent. Le complexe coloré est ensuite mesuré par spectrophotométrie. La méthode de référence définie par l'ICSH consiste à récupérer le surnageant d'une solution contenant du sérum et un agent de déprotéinisation (utilisé afin de dissocier le fer ferrique, Fe(III) lié aux protéines), auquel est ajoutée la solution du chromogène disodium-4,7diphényl-1,10-phénanthroline disulfonate (bathophénanthroline). Ce mélange est analysé par spectrophotométrie à $535 \mathrm{~nm}$ [15]. Il existe un très grand nombre de chromogènes. Les plus répandus sont :

- Férène : disodium 3-(2-pyridyl)-5,6-bis-[2-(5-furyle acide sulfonique)]-1,2,4-triazine.

- Ferrozine : 3 - (2-pyridyl) -5,6-bis- (5-phényle acide sulfonique)] -1,2,4- triazine.

- TPTZ : [2,4,6-tri-(2-pyridyl)-5-triazine].

Leurs longueurs d'onde respectives de lecture sont $562 \mathrm{~nm}, 593 \mathrm{~nm}$ et $595 \mathrm{~nm}$. Ce type de méthode est facile à mettre en œuvre, rapide et représente un faible coût. Le principal inconvénient de cette méthode est le risque d'une mauvaise estimation de la concentration en fer. En effet, les sources d'erreurs sont principalement au niveau du prétraitement initial des échantillons, notamment la dissociation incomplète du fer lié aux protéines, la coprécipitation du fer avec des protéines et la réduction incomplète du Fe(III) en Fe(II) [17]. De plus, la présence d'autres composés dans le sérum comme la bilirubine et les lipides peut créer des interférences spectrophotométriques $[18,19]$. Enfin, certains chromogènes utilisés, comme le ferrozine, réagissent également avec le cuivre. Cependant, à des concentrations physiologiques, cette erreur reste faible [15]. 
La spectrométrie d'absorption atomique est une technique où l'atome absorbe un quantum d'énergie lumineuse correspondant à une longueur d'onde spécifique et entraînant l'excitation de cet atome. La mesure de la quantité de lumière absorbée permet de déterminer la concentration de l'élément dans l'échantillon (loi de Beer-Lambert). L'échantillon est analysé après dilution et l'ajout d'un acide [20]. La longueur d'onde utilisée pour la détection du fer est de $248,3 \mathrm{~nm}$. C'est une technique robuste, facile à mettre en œuvre et spécifique qui permet également l'analyse d'autres métaux. Cependant, la gamme de travail est limitée, ce qui oblige une dilution des échantillons de concentrations élevées [21].

Afin de pallier les différentes contraintes rencontrées avec les méthodes de routine, la spectrométrie de masse couplée à une source plasma à courant induit (ICP-MS) peut être utilisée. Cette technique repose sur la détection d'ions mono-atomiques en analysant leur rapport masse sur charge. Cette méthode possède les avantages d'être multi élémentaire, d'avoir d'excellentes limites de détection (de l'ordre de $1 \mathrm{ng} \cdot \mathrm{L}^{-1}$ ) et de permettre la mesure de rapports isotopiques [22]. Un de ses inconvénients est l'existence d'interférences spectrales qui peuvent affecter la mesure : polyatomiques (par exemple, ${ }^{56} \mathrm{Fe}$ : ${ }^{40} \mathrm{Ar}^{16} \mathrm{O}^{+}$) et isobariques (par exemple, ${ }^{58} \mathrm{Fe}:{ }^{58} \mathrm{Ni}$ ). Ces interférences peuvent être en partie éliminées en utilisant une cellule de collision/réaction (CCT), un ICP-MS haute résolution ou des corrections mathématiques [23]. Plusieurs méthodes de quantification peuvent être utilisées : l'étalonnage externe, les ajouts dosés et la dilution isotopique [24-27]. L'étalonnage externe présente l'inconvénient de ne pas corriger les effets de matrices tandis que la méthode par ajouts dosés est complexe à mettre en œuvre. En associant la dilution isotopique (DI) à l'ICPMS, des déterminations exactes et précises peuvent être effectuées pour les éléments qui ont au moins deux isotopes libres d'interférences spectrales. La DI permet de compenser de nombreuses sources d'erreur, dont la perte d'échantillon pendant le processus de préparation. Un avantage majeur de cette approche par rapport aux procédures d'étalonnages classiques est qu'elle peut compenser des effets tels que les fluctuations du plasma, les instabilités de l'instrument et les pertes d'analytes [28]. Cette caractéristique est d'une grande importance pour la détermination de nombreux éléments dans des échantillons biologiques, où des effets de matrice importants sont couramment observés [22,28]. La DI-ICP-MS est ainsi utilisée pour analyser un grand nombre d'éléments comme le calcium $(\mathrm{Ca})$, le cuivre $(\mathrm{Cu})$, le potassium (K) dans le sérum humain $[22,29,30]$. D'autres matrices comme le sang, l'urine, le foie, le rein peuvent aussi être analysées [28].

Le Comité consultatif pour la quantité de matière (CCQM) du CIPM (Comité international des poids et mesures) considère la DI comme une méthode primaire pour la détermination de la teneur d'un élément [31]. L'incertitude globale peut être facilement déterminée sur la base de l'équation de mesure. Si elle est appliquée correctement, la DI peut directement relier le résultat de mesure au SI avec des incertitudes significativement plus petites
Tableau 1

Récapitulatif des informations relatives aux quatre échantillons étudiés.

\begin{tabular}{|c|c|c|}
\hline Échantillon & Type de matrice & $\begin{array}{c}\text { Concentration en } \\
\text { fer }\left(\mathbf{e n} \boldsymbol{\mu m o l} \cdot \mathbf{L}^{-1}\right)\end{array}$ \\
\hline $\begin{array}{c}\text { NIST SRM } \\
1598 \mathrm{a}\end{array}$ & $\begin{array}{c}\text { Sérum congelé, } \\
\text { d'origine animale }\end{array}$ & $30,1 \pm 1,1(k=2)$ \\
\hline $\begin{array}{c}\mathrm{ERM}^{\circledR} \text {-DA } \\
120 \mathrm{a}\end{array}$ & $\begin{array}{c}\text { Sérum congelé, } \\
\text { d'origine humaine }\end{array}$ & $9,5 \pm 0,4(k=2,2)$ \\
\hline $\mathrm{BCR}^{\circledR}-637$ & $\begin{array}{c}\text { Sérum congelé, } \\
\text { d'origine humaine }\end{array}$ & - \\
\hline $\mathrm{B} 17$ & $\begin{array}{c}\text { Sérum lyophilisé, } \\
\text { d'origine humaine }\end{array}$ & $24,4 \pm 0,8$ \\
\hline
\end{tabular}

que celles obtenues par d'autres méthodes. C'est pour cette raison que l'objectif du travail présenté dans cet article est le développement et la validation d'une méthode de référence pour le dosage du fer sérique par DI associée à l'ICP-MS.

\section{Matériels et méthodes analytiques mis en œuvre}

\section{1. Échantillons étudiés}

Les quatre échantillons analysés sont des sérums d'origines animale et humaine. Le premier échantillon est un matériau de référence certifié du NIST (SRM 1598a) constitué d'un sérum congelé d'origine animale (mélange de sérum bovin et porcin) certifié pour différents éléments dont le fer. Les trois autres échantillons sont des sérums d'origine humaine. L'échantillon « $\mathrm{ERM}^{\circledR}{ }^{\circledR}$ DA 120a Frozen human serum - trace metals » de l'institut national de métrologie du Royaume-Uni (LGC) est un sérum congelé certifié en cuivre $(\mathrm{Cu})$, sélénium $(\mathrm{Se})$ et zinc (Zn). L'échantillon « CRM BCR ${ }^{\circledR}-637$ Human serum » est un sérum congelé certifié en aluminium (Al), sélénium ( $\mathrm{Se}$ ) et zinc ( $\mathrm{Zn}$ ) par l'IRMM (Institute for Reference Materials and Measurements, UE). Le dernier échantillon «B17 » a été fourni par l'ANSM (Agence nationale de sécurité du médicament et des produits de la santé) à l'occasion d'un contrôle national de qualité des analyses de biologie médicale effectué en décembre 2012 [32]. Il se présente sous forme lyophilisée. Le tableau 1 récapitule les informations relatives aux échantillons utilisés.

Pour homogénéiser la présentation des concentrations en fer, l'unité utilisée est «la micromole par litre» $\left(\mu \mathrm{mol} \cdot \mathrm{L}^{-1}\right)$. Si besoin, la conversion de l'unité en «microgramme par litre, $\mu \mathrm{g} \cdot \mathrm{L}^{-1} \gg$ se fait en divisant par la masse molaire exacte du fer $\left(M_{\mathrm{Fe}}=55,845 \mathrm{~g} \cdot \mathrm{mol}^{-1}\right)$.

\subsection{Préparation des étalons}

La préparation des étalons se fait à partir d'un matériau de référence certifié de haute pureté pour le fer, BNM-001 (LNE), $(99,995 \pm 0,004) \%(k=2)$. Une masse d'environ $50 \mathrm{mg}$ est minéralisée dans un mélange de $2 \mathrm{~mL}$ d'eau Milli-Q ${ }^{\mathrm{TM}} 18,2 \mathrm{~m} \Omega \cdot \mathrm{cm}^{-1}$ (Millipore, Allemagne) et de $3 \mathrm{~mL}$ d'acide nitrique (Optima qualité, 
67-69 \%, $10 \mathrm{ng} \cdot \mathrm{L}^{-1} \mathrm{Fe}$, Fisher chemical, Canada). Après minéralisation complète, le volume total est complété jusqu'à $50 \mathrm{~mL}$ avec de l'eau Milli-Q ${ }^{\mathrm{TM}}$. Cette préparation est effectuée deux fois afin d'obtenir deux solutions mères de $1000 \mu \mathrm{g} \cdot \mathrm{g}^{-1}$ en fer chacune. Six étalons sont réalisés par pesée afin d'obtenir des solutions de concentration en fer comprise entre $0,5 \mathrm{ng} \cdot \mathrm{g}^{-1}$ et $20 \mathrm{ng} \cdot \mathrm{g}^{-1}$. Deux étalons marqués, enrichis en isotope ${ }^{57} \mathrm{Fe}$, sont utilisés : le premier sous la forme d'une solution marquée certifiée en ${ }^{57} \mathrm{Fe}$ à $(10,05 \pm 0,17) \mathrm{mg} \cdot \mathrm{kg}^{-1}\left(\right.$ Certipur ${ }^{\circledR}$ Reference Material Spike Solution, IRMM/Merck), le deuxième sous la forme métallique enrichie en ${ }^{57} \mathrm{Fe}$ (Eurisotop, France). Une quantité de $50 \mathrm{mg}$ de métal est mise en solution dans un mélange de $3 \mathrm{~mL}$ d' $\mathrm{H}_{2} \mathrm{O}, 2 \mathrm{~mL}$ d' $\mathrm{HNO}_{3}$ et $1 \mathrm{~mL}$ d'acide chlorhydrique (Optima qualité, 32-35 \%, $10 \mathrm{ng} \cdot \mathrm{L}^{-1} \mathrm{Fe}$, Fisher chemical, Canada). Les solutions obtenues sont d'environ $1000 \mu \mathrm{g} \cdot \mathrm{g}^{-1}$ en fer.

\subsection{Traitement des échantillons}

Quatre échantillons sont préparés à partir de sérums congelés (SRM 1598a, ERM ${ }^{\circledR}$-DA 120a, CRM BCR ${ }^{\circledR}$ _ 637) ou lyophilisé (B17). Pour les échantillons congelés, aucune étape de mise en solution préliminaire n'est nécessaire. Pour l'échantillon lyophilisé, B17, une étape de reconstitution est réalisée. Une quantité de $5 \mathrm{~mL}$ d'eau est ajoutée directement à la poudre. La préparation des échantillons doit tenir compte de la mise en œuvre de la dilution isotopique. L'étalon marqué doit être ajouté à l'échantillon le plus tôt possible lors du traitement. Toutes les étapes de préparation sont réalisées par gravimétrie jusqu'à l'ajout de l'étalon marqué. Une quantité identique en échantillon et en étalon marqué, d'environ $0,15 \mathrm{~g}$ à $0,30 \mathrm{~g}$, est mise en présence de $2 \mathrm{~mL}$ d' $\mathrm{HNO}_{3}($ Optima qualité) pour subir une digestion acide assistée d'un système de micro-ondes Discover SP-D (CEM, France). Les résidus de minéralisation sont ensuite repris dans $50 \mathrm{~mL}$ d'eau Milli- $\mathrm{Q}^{\mathrm{TM}}$ afin d'obtenir une concentration finale en fer comprise entre $5 \mathrm{ng} \cdot \mathrm{g}^{-1}$ et $15 \mathrm{ng} \cdot \mathrm{g}^{-1}$. Les échantillons obtenus sont analysés par ICP-MS.

Parallèlement, une évaluation préliminaire de la teneur en fer des flacons Pyrex ${ }^{\circledR}$ (CEM, France) utilisés pour la minéralisation est réalisée par ICP-MS. Pour cela, avant chaque analyse, une série de dix blancs, contenant $2 \%$ d' $\mathrm{HNO}_{3}$ dans $50 \mathrm{~mL}$ d'eau, est préparée selon le même protocole que pour les échantillons.

\subsection{Détection par ICP-MS}

La détection s'effectue avec un ICP-MS. Trois instruments de mesure différents ont été utilisés dans cette étude : Agilent 7700× ICP-Q-MS (Agilent Technologies, USA), iCAP Qc ICP-Q-MS (Thermo Fisher Scientific, Allemagne) et ELEMENT XR ${ }^{\mathrm{TM}}$ ICP-MS (Thermo Fisher Scientific, Allemagne). Des essais préliminaires ont permis d'optimiser les différentes configurations utilisées pour l'analyse du fer. Les deux premiers ICPMS sont de type quadripolaire avec une cellule de collision/réaction. La cellule est utilisée en mode réactionnel
Tableau 2

Paramètres de fonctionnement des instruments.

\begin{tabular}{|c|c|}
\hline \multicolumn{2}{|c|}{ Paramètres de l'Agilent 7700 $\times$ ICP-Q-MS } \\
\hline Paramètres du plasma & Paramètres d'acquisition \\
\hline $\begin{array}{l}\text { Puissance : } 1550 \mathrm{~W} \\
\text { Débit gaz vecteur : } 1 \mathrm{~L} \cdot \mathrm{min}^{-1} \\
\text { Débit gaz plasmagène }: 15 \mathrm{~L} \cdot \mathrm{min}^{-1} \\
\text { Débit gaz auxiliaire }: 1 \mathrm{~L} \cdot \mathrm{min}^{-1}\end{array}$ & $\begin{array}{l}\text { Isotopes mesurés : }{ }^{56} \mathrm{Fe}, \\
{ }^{57} \mathrm{Fe} \\
\text { Points par pics : } 3 \\
\text { Temps d'acquisition par } \\
\text { point }: 4 \mathrm{~s} \\
\text { Répétition : } 5\end{array}$ \\
\hline \multicolumn{2}{|c|}{ Paramètres de la cellule de collision/réaction } \\
\hline \multicolumn{2}{|c|}{$\begin{array}{l}\text { Débit } \mathrm{H}_{2}: 5 \mathrm{~mL} \cdot \mathrm{min}^{-1} \\
\text { Biais CCT }:-18 \mathrm{~V} \\
\text { Biais quadripôle }:-15 \mathrm{~V}\end{array}$} \\
\hline \multicolumn{2}{|c|}{ Paramètres de l'iCAP Qc ICP-Q-MS } \\
\hline Paramètres du plasma & Paramètres d'acquisition \\
\hline $\begin{array}{l}\text { Puissance : } 1550 \mathrm{~W} \\
\text { Débit gaz vecteur : } 1,05 \mathrm{~L} \cdot \mathrm{min}^{-1} \\
\text { Débit gaz plasmagène }: 14 \mathrm{~L} \cdot \mathrm{min}^{-1} \\
\text { Débit gaz auxiliaire }: 0,8 \cdot \mathrm{min}^{-1}\end{array}$ & $\begin{array}{l}\text { Isotopes mesurés : }{ }^{56} \mathrm{Fe}, \\
{ }^{57} \mathrm{Fe} \\
\text { Nombre de balayage }: 60 \\
\text { Dwell time }: 0,2 \mathrm{~s} \\
\text { Répétition : } 5\end{array}$ \\
\hline \multicolumn{2}{|c|}{ Paramètres de la cellule de collision/réaction } \\
\hline \multicolumn{2}{|c|}{$\begin{array}{l}\text { Débit } \mathrm{He}: 4 \mathrm{~mL} \cdot \mathrm{min}^{-1} \\
\text { Biais CCT }:-21 \mathrm{~V} \\
\text { Biais quadripôle }:-18 \mathrm{~V}\end{array}$} \\
\hline \multicolumn{2}{|c|}{ Paramètres de l'ELEMENT XR ${ }^{\mathrm{TM}}$ ICP-MS } \\
\hline Paramètres du plasma & Paramètres d'acquisition \\
\hline $\begin{array}{l}\text { Puissance : } 1550 \mathrm{~W} \\
\text { Débit gaz vecteur : } 1,06 \mathrm{~L} \cdot \mathrm{min}^{-1} \\
\text { Débit gaz plasmagène }: 16 \mathrm{~L} \cdot \mathrm{min}^{-1} \\
\text { Débit gaz auxiliaire : } 0,8 \mathrm{~L} \cdot \mathrm{min}^{-1}\end{array}$ & $\begin{array}{l}\text { Isotopes mesuré } \mathrm{s}:{ }^{56} \mathrm{Fe}, \\
{ }^{57} \mathrm{Fe} \\
\text { Temps d'intégration par } \\
\text { point }: 0,05 \mathrm{~s} \\
\text { Nombre de points par pic } \\
20 \\
\text { Nombre de balayage : } 15 \\
\text { Répétition : } 5\end{array}$ \\
\hline \multicolumn{2}{|l|}{ Paramètres haute résolution } \\
\hline $\begin{array}{l}\text { Résolution : } 4500 \\
\text { Masse de l'aimant : 55,934 }\end{array}$ & \\
\hline
\end{tabular}

avec un débit d'hydrogène de $5 \mathrm{~mL} \cdot \mathrm{min}^{-1}$ pour l'instrument Agilent 7700×. Pour l'instrument iCAP Qc, la cellule est utilisée en mode collision avec un débit d'hélium de $4 \mathrm{~mL} \cdot \mathrm{min}^{-1}$. L'instrument ELEMENT XR est un instrument à secteur magnétique utilisé en moyenne résolution $(m / \Delta m \approx 4500)$. Les différentes caractéristiques des instruments sont récapitulées dans le tableau 2.

Pour les trois instruments, les réglages des débits de gaz, la position de la torche ainsi que celle de la lentille ionique sont optimisés pour chaque analyse afin d'obtenir une sensibilité maximale sur une solution multiélémentaire à $1 \mu \mathrm{g} \cdot \mathrm{L}^{-1}$.

\subsection{Dilution isotopique}

La DI consiste à déterminer la concentration d'un élément (fer) dans un échantillon par l'ajout d'une quantité connue d'un étalon marqué de ce même élément dont la composition isotopique a été artificiellement modifiée. Une fois l'équilibre isotopique atteint, les rapports 


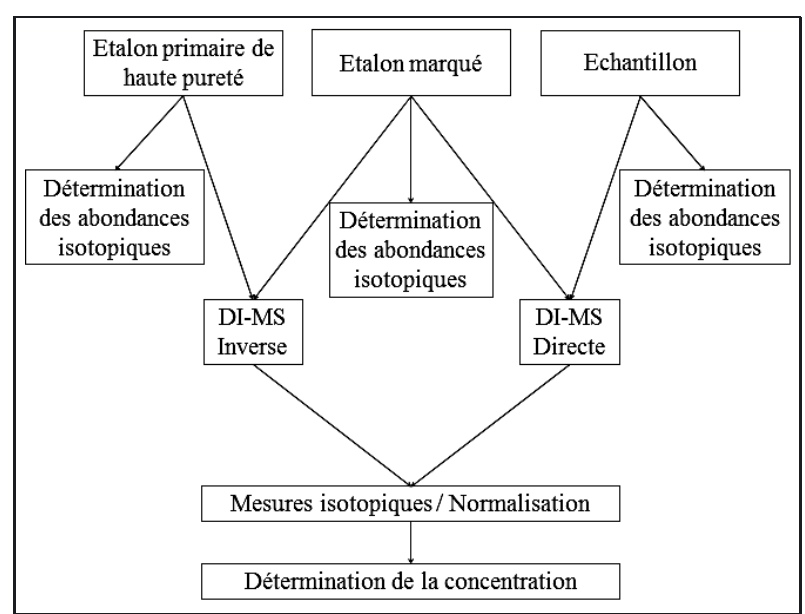

Fig. 1. - Différentes étapes de réalisation de la dilution isotopique utilisée comme méthode primaire.

isotopiques sont mesurés par spectrométrie de masse élémentaire [33]. Il est nécessaire de bien choisir le rapport isotopique mesuré pour mener la DI. On privilégie de préférence deux isotopes libres d'interférents et ayant une abondance naturelle élevée.

Pour cette étude, nous avons choisi le rapport isotopique ${ }^{56} \mathrm{Fe} /{ }^{57} \mathrm{Fe}$. Afin d'assurer le caractère primaire de la méthode, une approche métrologique de la DI est par ailleurs nécessaire. Le protocole fait intervenir plusieurs étapes qui sont synthétisées dans la figure 1 .

Il faut déterminer les abondances isotopiques de l'échantillon, de l'étalon marqué (sp) et de l'étalon de haute pureté (eta). Ensuite, la concentration de l'étalon marqué $\left(C_{\mathrm{sp}}, \mu \mathrm{g} \cdot \mathrm{g}^{-1}\right)$ est calculée à partir de la mesure du rapport des deux isotopes A et B (respectivement fer-56 et fer-57) dans le mélange entre l'étalon primaire de haute pureté et l'étalon marqué en utilisant l'équation (1); cette étape est la DI inverse.

$$
C_{\mathrm{sp}}=\frac{m_{\mathrm{eta}} C_{\mathrm{eta}}}{m_{\mathrm{spi}}}\left[\frac{R_{i} K_{i}(B)_{\mathrm{eta}}-(A)_{\mathrm{eta}}}{(A)_{\mathrm{sp}}-R_{i} K_{i}(B)_{\mathrm{sp}}}\right]
$$

avec :

$m_{\text {eta }}(\mathrm{g}), \quad$ prise d'essai de la solution étalon primaire ;

$m_{\mathrm{sp}}(\mathrm{g}), \quad$ masse d'étalon marqué pour la dilution isotopique inverse ;

$C_{\text {eta }}\left(\mu \mathrm{g} \cdot \mathrm{g}^{-1}\right)$, concentration de la solution étalon primaire ;

$C_{\mathrm{sp}}\left(\mu \mathrm{g} \cdot \mathrm{g}^{-1}\right)$, concentration de l'élément dans la solution de l'étalon marqué ;

$(A)_{\text {eta }}(\%), \quad$ abondance naturelle de l'isotope A dans l'étalon primaire ;

$(B)_{\text {eta }}(\%)$, abondance naturelle de l'isotope B dans l'étalon primaire ;

$(A)_{\mathrm{sp}}(\%), \quad$ abondance de l'isotope A dans l'étalon marqué ;
$(B)_{\mathrm{sp}}(\%), \quad$ abondance de l'isotope B dans l'étalon marqué ;

$R_{\mathrm{i}}(-), \quad$ rapport isotopique pour la dilution isotopique inverse ;

$K_{\mathrm{i}}(-), \quad$ facteur de correction de dérive et de biais en masse pour la dilution inverse.

La concentration de l'élément à doser ( $C_{\text {éch }}$ en $\mu \mathrm{g} \cdot \mathrm{g}^{-1}$ ) est calculée avec l'équation (2) à partir de la mesure du rapport des isotopes A et B dans le mélange échantillon-étalon marqué; cette étape est la DI directe.

$$
C_{\mathrm{ech}}=\frac{m_{\mathrm{spd}} C_{\mathrm{sp}}\left[\frac{R_{\mathrm{d}} K_{\mathrm{d}}(B)_{\mathrm{sp}}-(A)_{\mathrm{sp}}}{(A)_{\mathrm{ech}}-R_{\mathrm{d}} K_{\mathrm{d}}(B)_{\mathrm{ech}}}\right]}{m_{\mathrm{ech}}} d
$$

avec :

$m_{\text {spd }}(\mathrm{g}), \quad$ masse d'étalon marqué pour la dilution isotopique directe ;

$m_{\text {ech }}(\mathrm{g}), \quad$ prise d'essai de l'échantillon;

$C_{\text {sp }}\left(\mu \mathrm{g} \cdot \mathrm{g}^{-1}\right)$, concentration de l'élément dans la solution de l'étalon marqué;

$C_{\text {ech }}\left(\mu \mathrm{g} \cdot \mathrm{g}^{-1}\right)$, concentration de l'élément dans l'échantillon;

$(A)_{\mathrm{sp}}(\%), \quad$ abondance de l'isotope A dans l'étalon marqué ;

$(B)_{\mathrm{sp}}(\%), \quad$ abondance de l'isotope B dans l'étalon marqué ;

$(A)_{\text {ech }}(\%), \quad$ abondance naturelle de l'isotope A dans l'échantillon;

$(B)_{\text {ech }}(\%), \quad$ abondance naturelle de l'isotope B dans l'échantillon;

$R_{\mathrm{d}}(-), \quad$ rapport isotopique pour la dilution isotopique directe;

$K_{\mathrm{d}}(-), \quad$ facteur de correction de dérive et de biais en masse pour la dilution directe;

$d\left(\mathrm{~g} \cdot \mathrm{mL}^{-1}\right), \quad$ masse volumique de l'échantillon.

Une normalisation des mesures des $R_{\mathrm{A} / \mathrm{B}}$ est effectuée pour prendre en compte le biais en masse du spectromètre de masse. L'analyse d'un point de contrôle préparé à partir de l'étalon de haute pureté permet de faire cette correction [34]. Enfin, la concentration de l'échantillon est déterminée en combinant les équations (1) et (2).

\section{6. Évaluation des incertitudes}

La traçabilité des résultats de mesure ainsi que leur comparabilité ne peuvent pas être assurées si une incertitude ne leur est pas associée. La démarche retenue pour ce travail repose sur la méthode analytique recommandée par le Guide pour l'expression des incertitudes 
Tableau 3

Isotopie mesurée pour l'étalon de fer d'Eurisotop.

\begin{tabular}{|c|c|c|c|c|}
\hline & ${ }^{\mathbf{5 4}} \mathbf{F e}$ & ${ }^{\mathbf{5 6}} \mathbf{F e}$ & ${ }^{\mathbf{5 7}} \mathbf{F e}$ & ${ }^{\mathbf{5 8}} \mathbf{F e}$ \\
\hline $\begin{array}{c}\text { Abondance } \\
\text { en \% } \\
(\boldsymbol{k}=\mathbf{2})\end{array}$ & $0,0014 \pm$ & $1,39 \pm$ & $96,63 \pm$ & $1,97 \pm$ \\
0,027 & 0,36 & 0,40 & 0,24 \\
\hline $\begin{array}{c}\text { Valeurs } \\
\text { Eurisotop } \\
\text { fournies } \\
(\boldsymbol{\%})\end{array}$ & 0,03 & 1,65 & 96,28 & 2,04 \\
\hline
\end{tabular}

(GUM) [35]. L'incertitude est calculée par la loi de propagation des incertitudes à partir du modèle mathématique décrivant l'ensemble du processus de mesure. Le modèle mathématique utilisé est l'équation générale (1) ou (2) de la dilution isotopique. Les incertitudes ont été calculées à partir du logiciel Wincert V.3.11.2002.0115 (Implex développé au LNE).

\section{Résultats}

L'objectif est de développer et de valider une méthode primaire pour le dosage du fer dans du sérum. Cette méthode doit permettre un dosage fiable pour des concentrations physiologiques comprises entre $500 \mu \mathrm{g} \cdot \mathrm{L}^{-1}$ et $1700 \mu \mathrm{g} \cdot \mathrm{L}^{-1}$ [5]. Cette méthode de référence permettra d'assigner des valeurs de référence traçables aux matériaux d'étalonnage et aux échantillons de contrôle de qualité, et aussi d'évaluer la justesse des méthodes de routine. Un protocole métrologique de la dilution isotopique associée à l'ICP-MS (DI-ICP-MS) a été mis en place pour atteindre cet objectif.

\subsection{Caractérisation de l'étalon marqué}

Pour s'assurer de la traçabilité des résultats de mesure, des étalons certifiés en isotopie et en teneur ont été utilisés. Et l'étalon marqué ${ }^{57} \mathrm{Fe}$ d'Eurisotop (laboratoire non LNM) n'ayant pas été certifié suivant un protocole métrologique reconnu, a été caractérisé. Il a été analysé une fois par mois, pendant six mois, en utilisant les ICP-MS ELEMENT XR et iCAP Qc. Une moyenne des valeurs obtenues $(n=6)$ a été calculée. Un test de Cochran est réalisé afin d'éliminer les éventuelles valeurs aberrantes de manière statistique. L'incertitude élargie est choisie avec un facteur d'élargissement, $k$, de 2 . Les valeurs obtenues sont compatibles avec celles données par le producteur du métal enrichi (tab. 3). Ceci indique que l'isotopie de ce matériau est stable.

\section{2. Évaluations préliminaires des concentrations en fer}

Afin d'évaluer la gamme de concentrations dans laquelle se situent les échantillons et d'adapter, en conséquence, la dilution isotopique aux échantillons, une estimation préliminaire de la concentration des échantillons en élément fer est effectuée avec les ICP-MS ELEMENT XR et iCAP Qc à l'aide d'un étalonnage
Tableau 4

Concentrations en fer de l'échantillon « NIST SRM 1598a » obtenues avec différents instruments.

\begin{tabular}{|c|c|c|c|}
\hline & $\begin{array}{c}\text { ICP-Q-MS } \\
\left(\mathbf{H}_{2}\right)\end{array}$ & $\begin{array}{c}\text { ELEMENT } \\
\mathbf{X R}^{\text {TM }}\end{array}$ & $\begin{array}{c}\text { ICP-Q-MS } \\
(\mathbf{H e})\end{array}$ \\
\hline $\begin{array}{c}\text { Concentration } \\
\text { en fer } \\
\left(\boldsymbol{\mu g} \cdot \mathbf{L}^{-\mathbf{1}}, \boldsymbol{k}=\mathbf{2}\right)\end{array}$ & $1653 \pm 38$ & $1629 \pm 46$ & $1632 \pm 41$ \\
\hline Biais (\%) & -2 & -3 & -3 \\
\hline $\begin{array}{c}\text { Écart } \\
\text { normalisé } \\
\left(\boldsymbol{E}_{\mathbf{N}}\right)\end{array}$ & 0,76 & 1,35 & 1,28 \\
\hline
\end{tabular}

externe puis en réalisant des ajouts dosés sur l'échantillon, pour une estimation plus précise (valeurs non reportées).

De plus, l'évaluation de la contamination éventuelle en fer des tubes de minéralisation donne une concentration moyenne de $10 \mu \mathrm{g} \cdot \mathrm{L}^{-1}$. Les tubes de minéralisation ayant une concentration en fer supérieure à cette moyenne sont écartés.

\subsection{Validation de la méthode}

La validation de la méthode (justesse et incertitude) a été réalisée par l'analyse de l'échantillon «SRM 1598a» et en participant à une comparaison interlaboratoire. Une série de quatre réplicats du matériau SRM 1598a est préparée et analysée sur les trois instruments de mesure afin de s'assurer qu'il n'y a pas d'impact sur le résultat final. La teneur en fer certifiée par le NIST est de (1 $680 \pm 60) \mu \mathrm{g} \cdot \mathrm{L}^{-1}(k=2)$ avec une masse volumique d'échantillon de $(1,0274 \pm 0,0006) \mathrm{g} \cdot \mathrm{mL}^{-1}$. Les résultats reportés dans le tableau 4 sont les valeurs moyennes des quatre réplicats pour chaque instrument de mesure avec leurs incertitudes élargies de répétabilité $(k=2)$.

Les valeurs mesurées peuvent être comparées à la valeur certifiée en utilisant l'écart normalisé $E_{\mathrm{N}}$ [36].

$$
E_{\mathrm{N}}=\left|\frac{C_{\text {éch }}-C_{\text {cert }}}{\sqrt{u_{\text {Céch }}^{2}+u_{\text {Ccert }}^{2}}}\right|
$$

avec :

$C_{\text {éch }}$ valeur déterminée ;

$C_{\text {cert }}$, valeur certifiée ;

$u_{\text {Céch}}$, incertitude-type de $C_{\text {éch }}$;

$u_{\text {Ccert }}$, incertitude-type de $C_{\text {cert }}$.

Les écarts normalisés obtenus avec les trois instruments sont inférieurs à 2 , ce qui signifie que les valeurs mesurées ne sont pas statistiquement significativement différentes de la valeur de référence. La méthode développée est donc validée par l'analyse de ce matériau de référence certifié. L'intérêt d'avoir travaillé avec 


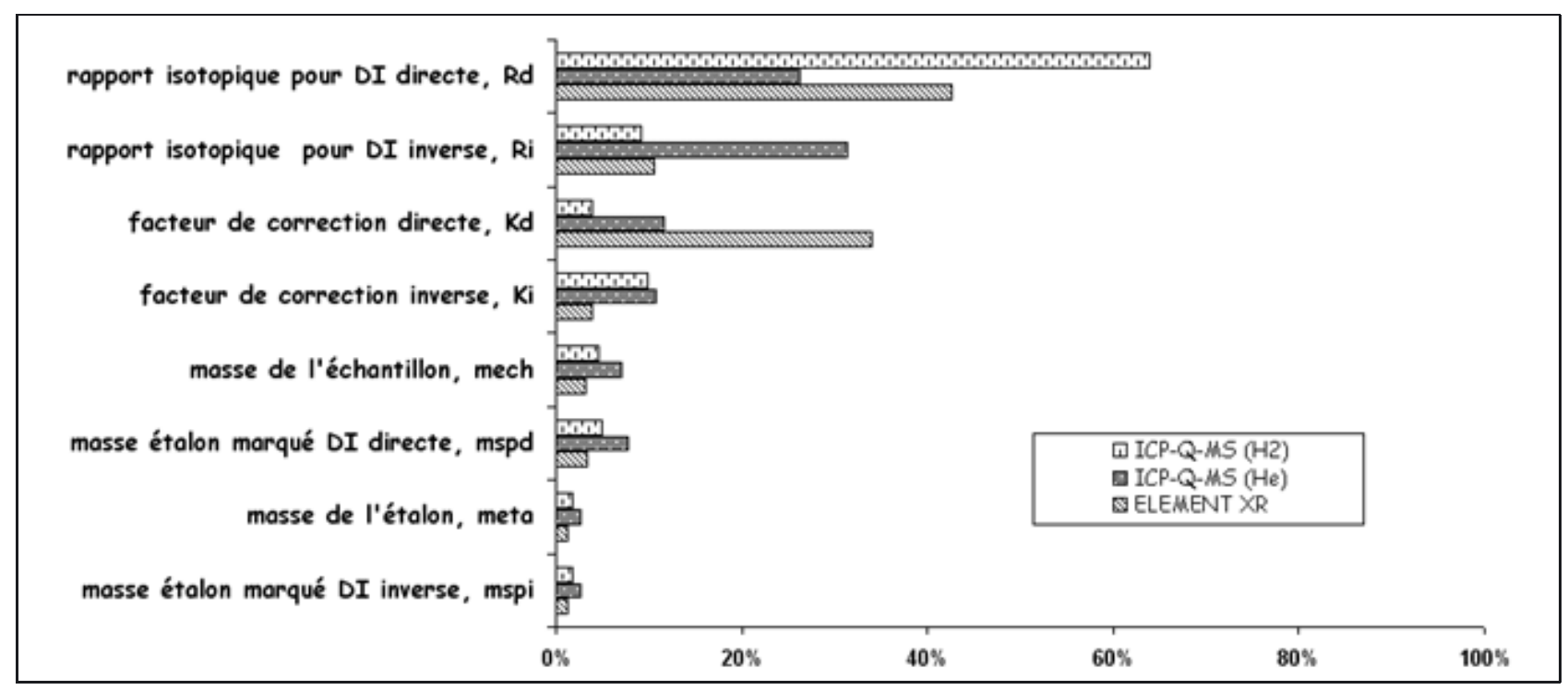

Fig. 2. - Répartition des composantes d'incertitude sur la détermination de la concentration en fer au LNE.

trois instruments de mesure est de pouvoir comparer leurs performances. En effet, ils utilisent des approches différentes pour corriger les interférences, qui dans le cas du fer sont principalement spectrales de type polyatomique avec l'argon et le calcium présent, d'une manière générale, dans le sérum avec une concentration de l'ordre de $100 \mathrm{mg} \cdot \mathrm{L}^{-1}[37]$ :

$$
\begin{aligned}
& { }^{56} \mathrm{Fe}:{ }^{40} \mathrm{Ar}^{16} \mathrm{O}^{+}(R=2500),{ }^{40} \mathrm{Ca}^{16} \mathrm{O}^{+}(R=2480) ; \\
& { }^{57} \mathrm{Fe}:{ }^{40} \mathrm{Ar}^{16} \mathrm{OH}^{+}(R=2000),{ }^{38} \mathrm{Ar}^{18} \mathrm{OH}^{+}(R= \\
& 2000),{ }^{40} \mathrm{Ca}^{16} \mathrm{OH}^{+}(R=2100)[38] .
\end{aligned}
$$

Une deuxième validation a été effectuée lors de la participation du LNE à la comparaison clé organisée dans le cadre du CCQM (comparaison CCQM-K107) pilotée en 2013 par le LGC sur l'échantillon « ERM ${ }^{\circledR}$ DA 120a ». La méthode de quantification retenue par tous les laboratoires est la DI-ICP-MS : six ont utilisé l'ICP$\mathrm{MS}$ à secteur magnétique et quatre, l'ICP-MS quadripôle avec une cellule de collision (CCT). L'étape de préparation d'échantillon choisie par la majorité des participants est une digestion acide avec activation par champ micro-ondes. Seulement deux laboratoires ont réalisé une simple dilution du sérum et ils obtiennent les valeurs les plus faibles. En effet, la digestion acide permet d'extraire la totalité du fer présent dans le sérum. Avec une dilution du sérum il y a un risque de précipitation des protéines et donc une sous estimation de la teneur en fer. Les résultats obtenus sur l'échantillon ERM ${ }^{\circledR}$-DA 120a, ayant une densité de $(1,0226 \pm 0,0203) \mathrm{kg} \cdot \mathrm{L}^{-1}$, sont représentés dans le tableau 5.

Une comparaison des moyennes entre les résultats du LNE et la valeur de référence KCRV conduit à un écart normalisé inférieur à 2 , ce qui montre la justesse de la méthode développée et l'absence de biais. On peut noter que la concentration en fer du sérum ERM ${ }^{\circledR}$-DA 120a est environ trois fois moins élevée que celle du sérum SRM 1598a. L'incertitude relative obtenue est de l'ordre
Tableau 5

Résultats de la comparaison CCQM-K107 (échantillon ERM $^{\circledR}$-DA 120a).

\begin{tabular}{|c|c|c|c|}
\hline & $\begin{array}{c}\text { Valeur de } \\
\text { référence } \\
\text { mesurée }\end{array}$ & Valeur $\mathrm{KCRV}^{*}$ & $\begin{array}{c}\text { Écart } \\
\text { normalisé } \\
\left(E_{\mathrm{N}}\right)\end{array}$ \\
\hline $\begin{array}{c}\text { Concentration } \\
\text { en fer } \\
\left(\mu \mathrm{g} \cdot \mathrm{L}^{-1}\right)\end{array}$ & $\begin{array}{c}541 \pm 22 \\
(k=2)\end{array}$ & $\begin{array}{c}530 \pm 22 \\
(k=2)\end{array}$ & $0,74<2$ \\
\hline
\end{tabular}

*Valeur de référence de la comparaison.

de $4 \%$, ce qui est inférieur à l'incertitude cible de la comparaison $(5 \%)$.

La méthode développée a été ainsi validée pour un domaine de concentrations en fer comprises entre $510 \mu \mathrm{g} \cdot \mathrm{L}^{-1}$ et $2050 \mu \mathrm{g} \cdot \mathrm{L}^{-1}$ (soit $9 \mu \mathrm{mol} \cdot \mathrm{L}^{-1}$ à $\left.37 \mu \mathrm{mol} \cdot \mathrm{L}^{-1}\right)$ avec une incertitude élargie $(k=2)$ inférieure à $5 \%$ sur l'ensemble du domaine.

\section{4. Évaluation de l'incertitude de mesure de la concentration en fer}

Une évaluation des principales sources d'incertitude est effectuée. La figure 2 montre un exemple de la répartition des composantes d'incertitude pour une mesure individuelle.

Les principales sources d'incertitude sont les rapports isotopiques inverses $\left(R_{\mathrm{i}}\right)$ et directs $\left(R_{\mathrm{d}}\right)$. La tendance reste sensiblement la même suivant le type d'ICP-MS utilisé. L'effet sur l'incertitude finale des rapports isotopiques directs $\left(R_{\mathrm{d}}\right)$ a tendance à être plus élevé que celui des rapports isotopiques inverses $\left(R_{\mathrm{i}}\right)$. En effet, le type d'échantillon est différent puisque pour la DI directe, l'échantillon est le sérum donc une matrice complexe, tandis que pour la DI inverse, l'échantillon est une solution d'un étalon de haute pureté. D'autres facteurs ont un effet mineur 
Tableau 6

Concentrations en fer sur le BCR ${ }^{\circledR}-637$ et le SRM 1598a.

\begin{tabular}{|c|c|c|c|}
\hline & $\begin{array}{c}\text { BCR } \\
(n=4)\end{array}$ & $\begin{array}{c}\text { SRM 1598a } \\
(1680 \pm 60) \\
\mu \mathrm{g} \cdot \mathrm{L}^{-1}\end{array}$ & $\begin{array}{c}\text { Écart } \\
\text { normalisé }\left(E_{\mathrm{N}}\right) \\
\text { sur le SRM }\end{array}$ \\
\hline $\begin{array}{c}\text { Concentration } \\
\text { en fer } \\
\left(\mu \mathrm{g} \cdot \mathrm{L}^{-1}, k=2\right)\end{array}$ & $1671 \pm 74$ & $1174 \pm 43$ & $0,19<2$ \\
\hline
\end{tabular}

sur l'incertitude finale notamment les facteurs de correction de dérive et de biais en masse $\left(K_{\mathrm{d}}\right.$ et $\left.K_{\mathrm{i}}\right)$ ainsi que les différentes prises d'essai ( $\left.m_{\mathrm{ech}}, m_{\mathrm{spd}}, m_{\mathrm{spi}}, m_{\mathrm{eta}}\right)$. Tous ces facteurs correspondent au calcul de l'incertitude pour une mesure. Cependant, lorsque la valeur finale est calculée sur la base de plusieurs réplicats, la fidélité doit être prise en compte. La fidélité est l'étroitesse de l'accord entre les valeurs mesurées obtenues par des mesurages répétés [13]. Ce facteur se calcule en divisant l'écart type par la racine carrée du nombre de réplicats. Plus la dispersion des résultats est élevée, plus la fidélité sera grande. Dans ce cas, cette variable est largement prépondérante par rapport aux autres données.

\subsection{Application de la méthode à des échantillons inconnus}

Une fois la validité de la méthode démontrée dans l'intervalle de concentrations et aux incertitudes cibles, elle a été appliquée à la caractérisation d'échantillons inconnus afin d'assurer la mise en œuvre de programmes d'assurance qualité ou de contrôle qualité pour les laboratoires d'analyse biomédicale.

La méthode est ici appliquée à deux échantillons dont la teneur en fer était inconnue : le $\mathrm{BCR}^{\circledR}-637$, un matériau de référence certifié mais pas pour le fer, et à un échantillon fourni par l'ANSM lors d'un contrôle national de qualité (CNQ) effectué en 2012 (échantillon B17).

\subsubsection{Echantillon $B C R^{\circledR}-637$}

L'échantillon $\mathrm{BCR}{ }^{\circledR}-637$ a été analysé uniquement par l'ICP-MS ELEMENT XR ${ }^{\mathrm{TM}}$. Afin de contrôler la justesse de l'analyse, l'échantillon SRM 1598a a été utilisé comme point de contrôle. Cet échantillon est inséré dans la séquence d'analyse après chaque échantillon du BCR. Le tableau 6 montre les résultats obtenus.

Il faut noter qu'il n'y a pas eu de biais de mesure lors de l'analyse, au regard du résultat obtenu pour le SRM. En effet, l'écart normalisé est largement inférieur à 2, ce qui indique la justesse de la mesure. La valeur obtenue est comprise dans la gamme des valeurs physiologiques. En outre, l'incertitude finale est acceptable très correcte puisqu'elle est de l'ordre de $4 \%$.

\subsection{2. Échantillon B17}

Pour le dosage du fer dans cet échantillon, 1472 laboratoires ont participé au contrôle national de qualité

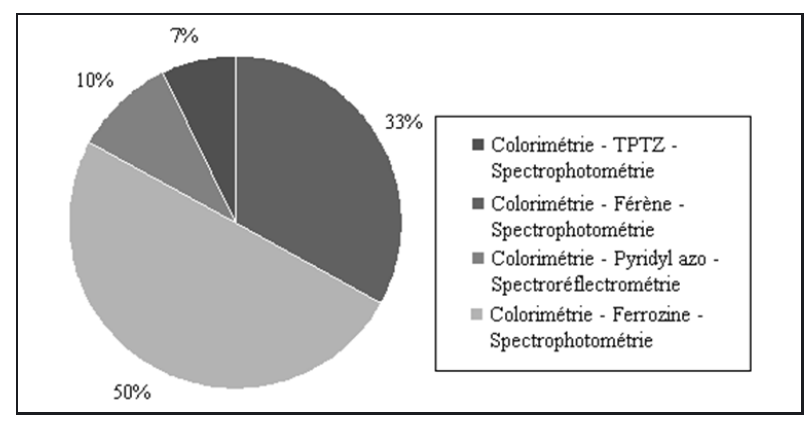

Fig. 3. - Répartition des méthodes de routine utilisées lors du CNQ ANSM 2012 pour la mesure du fer.

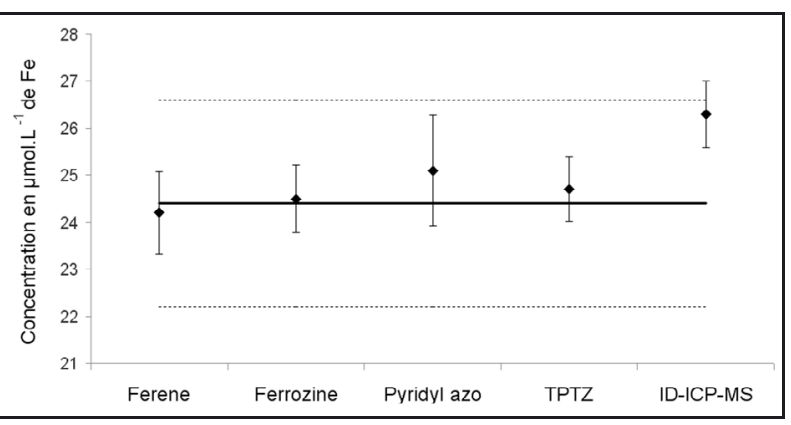

Fig. 4. - Résultats du CNQ de 2012 (sur l'échantillon B17). Les valeurs sont représentées avec les écarts types associés. La valeur consensuelle est indiquée par le trait plein. Les limites acceptables de la valeur consensuelle sont délimitées en pointillés.

(CNQ) organisé par l'ANSM en 2012. Tous les laboratoires participants ont utilisé une méthode colorimétrique. Environ $90 \%$ d'entre eux ont associé la méthode colorimétrique à une détection spectrophotométrique (fig. 3). Le chromogène le plus représenté était la ferrozine, utilisé par la moitié des laboratoires (fig. 3).

La valeur consensuelle, moyenne de l'ensemble des résultats des laboratoires après élimination des valeurs aberrantes est de $(1363 \pm 45) \mu \mathrm{g} \cdot \mathrm{L}^{-1}$.

La valeur déterminée par la méthode développée

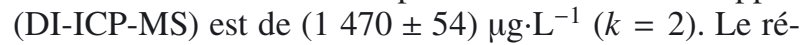
sultat obtenu est une moyenne des valeurs déterminées à l'aide de l'instrument ELEMENT XR ainsi que de l'instrument iCAP Qc. Cette valeur est comparée aux concentrations déterminées par les autres laboratoires (fig. 4).

La valeur obtenue par la méthode primaire développée est comprise entre les limites acceptables définies par l'ANSM. Cependant cette valeur déterminée par DI-ICPMS est plus élevée (entre $5 \%$ et $8 \%$ ) que les valeurs déterminées par les méthodes utilisées par les différents laboratoires. En effet, les méthodes de dosage utilisées sont différentes étant donné que les laboratoires ont effectué des dosages colorimétriques. La colorimétrie présente comme principal inconvénient de nécessiter une étape de pré-traitement des échantillons pour les analyser par spectrophotométrie. Cette étape consiste à dissocier le fer ferrique lié aux protéines et à le réduire en fer ferreux 
Tableau 7

Résumé des résultats obtenus sur les différents échantillons exprimés dans les mêmes unités.

\begin{tabular}{|c|c|c|}
\hline & $\begin{array}{c}\text { Concentration en fer } \\
\left(\text { en } \mu \mathrm{mol} \cdot \mathrm{L}^{-1}\right) \\
\text { et incertitudes } \\
\text { élargies }(\text { en } \%)\end{array}$ & $\begin{array}{c}\text { Valeurs } \\
\text { physiologiques } \\
\text { en } \mu \mathrm{mol} \cdot \mathrm{L}^{-1}\end{array}$ \\
\hline NIST SRM 1598a & $29,3(3)$ & \multirow{2}{*}{$10-30$} \\
\cline { 1 - 2 } ERM $^{\circledR}$-DA 120a & $9,7(4)$ & \\
\hline BCR $^{\circledR}$-637 & $21(4)$ & \\
\hline B17 $^{\circledR}$ & $26,3(3,5)$ & \\
\hline
\end{tabular}

pour que le complexe fer-chromogène puisse avoir lieu. Donc, il y a le risque d'une dissociation incomplète du fer lié aux protéines, d'une co-précipitation du fer avec des protéines ainsi que de la réduction incomplète du $\mathrm{Fe}$ (III) en $\mathrm{Fe}(\mathrm{II})$ qui pourrait expliquer ce biais. Or, dans la méthode développée tout le fer, quelque soit sa forme chimique, est dissocié des protéines par la digestion acide assistée par champs micro-ondes. Le biais observé peut non seulement provenir des performances des méthodes mais d'un effet de matrice susceptible de varier d'une méthode à une autre sachant que ce dernier est minimisé par l'utilisation de la DI.

\section{Conclusion}

Le développement d'une méthode primaire de référence basée sur la mise en œuvre de la dilution isotopique (DI) associée à l'ICP-MS pour la détermination du fer total dans du sérum s'est montrée concluante. En effet, le protocole analytique a été validé par l'analyse d'un matériau de référence certifié (SRM 1598a) ainsi que par la participation à la comparaison clé CCQM-K107.

La traçabilité au SI des résultats obtenus a été établie à travers l'utilisation d'un étalon primaire certifié en fer (BNM-001), par la mise en œuvre de protocoles métrologiques durant toutes les étapes de la préparation des échantillons et par l'établissement d'un bilan complet de l'incertitude de mesure.

L'application de cette méthode à d'autres échantillons de sérum $\left(\mathrm{BCR}^{\circledR}{ }^{\circledR}-637\right.$ et échantillon du contrôle de national de qualité ANSM B17) a démontré la validité de la méthode sur la mesure du fer pour des concentrations dans la gamme des valeurs physiologiques avec des incertitudes relatives inférieures à $5 \%$ ainsi que la faisabilité de l'application de la méthode pour l'assignation de valeurs de référence lors de programmes de contrôle qualité (tab. 7).

Cette méthode fera prochainement l'objet d'une demande d'accréditation du LNE selon les référentiels ISO EN 17025 et ISO EN 15189.

La méthode développée pourra ainsi être transférée sur d'autres éléments essentiels comme par exemple des éléments d'importance en biologie médicale tels que les électrolytes ( $\mathrm{Na}, \mathrm{Mg}, \mathrm{K}$ ) ou sur d'autres matrices comme le sang (métaux lourds tels que le plomb).

\section{Références}

[1] Abbaspour N., Hurrell R. et Kelishadi R., "Review on iron and its importance for human health", J. Res. Med. Sci., 19, 2014, 164-174.

[2] Del Castillo M.E., Montes Gayón M. et SanzMEDEL A., "The potential of mass spectrometry to study iron-containing proteins used in clinical diagnosis", Analytica Chimica Acta, 634, 2009, 1-14.

[3] TRUMBo P., "Dietary reference intakes: vitamin A, vita$\min \mathrm{K}$, arsenic, boron, chromium, copper, iodine, iron, manganese, molybdenum, nickel, silicon, vanadium, and zinc", J. Am. Diet. Assoc., 101, 2001, 294-301.

[4] ARORA S. et KAPOOR R.K., "Iron Metabolism in Humans: An Overview", INTECH, 2012, ISBN 978-95351-0605-0.

[5] Worwood M., "The laboratory assessment of iron status - an update", Clinica Chimica Acta, 259, 1997, 3-23.

[6] Rockey D.C. et CELlo J.P., "Evaluation of the gastrointestinal tract in patients with iron-deficiency anemia", Gastrointestinal tract and iron-deficiency anemia, 329, 1993, 1691-1695.

[7] Cohen-Solal A., Leclerce C., MebazaA A., De Groote P., DAmy T., ISNARD R. et Galinier M., "Diagnosis and treatment of iron deficiency in patients with heart failure: Expert position paper from French cardiologists", Archive of Cardiovascular Disease., 107, 2014, 563-571.

[8] «L'hémochromatose - hémochromatose héréditaire », Orphanet, Octobre 2006. https://www.orpha.net/data/ patho/Pub/fr/hemochromatose-FRfrPub92.pdf

[9] KANWAR P. et KowDlEy K.V., "Metal Storage Disorders: Wilson Disease and Hemochromatosis", Medical Clinics of North America, 98, 2014, 87-102.

[10] LeITMAN S.F., "Hemochromatosis : the new blood donor", Hematology ASH, 2013, 2013, 645-650.

[11] http://www.ameli.fr/l-assurance-maladie/ statistiques-etpublications/donnees-statistiques/ actes-de-biologie-medicale/biolam-2012-2014.php

[12] NF EN ISO 15189, 2012, «Laboratoires de biologie médicale-Exigences concernant la qualité et la compétence ».

[13] JCGM 200:2012 (E/F), $3^{e}$ édition, "International vocabulary of metrology - Basic and general concepts and associated terms (VIM) / Vocabulaire international de métrologie - Concepts fondamentaux et généraux et termes associés (VIM) », accessible par le site web du BIPM : http://www.bipm.org/fr/publications/guides/.

[14] http://www.bipm.org/jctlm/.

[15] ICSH, "Recommendations for the measurements of the serum iron in human blood", British Journal of Haematology, 38, 1978, 291-294.

[16] TAYLOR A., "Atomic spectrometry and the clinical chemistry of trace elements", J. Anal. At. Spectrom., 21 2006, 381-383. 
[17] TIETZ N.W., RINKER A.D. et MORRISON S.R., "When is a serum iron really a serum iron? The status of serum iron measurements", Clin. Chem., 40, 1994, 546-551.

[18] DING Y.S., LIU L.L., MA Y.F., LIN B.C., "Determination of total Iron binding capacity of serum by Capillary Electrophoresis", Chromatographia, 49, 1999, 71-74.

[19] MohSEN U.A., Elaish K.A. et Issa M., "Determination of iron in blood serum by spectrophotometric and atomic absorption methods as a comparative study", Cukurova Medical Journal, 38, 2013, 358-364.

[20] PerkinElmer, "Analytical methods for Atomic Absorption Spectroscopy", 1996.

[21] LEE K.T. et JACOB E., "Determination of serum iron and zinc by atomic absorption spectroscopy", Mikrochim. Acta., 1, 1974, 65-72.

[22] SARIEgo Muniz C., Marchante Gayón J.M., García AlONSO J.I., SANZ-MEDEL A., "Accurate determination of iron, copper and zinc in human serum by isotope dilution analysis using double-focusing ICP-MS", J. Anal. At. Spectrom., 14, 1999, 1505-1510.

[23] Vanhaecke F., Balcaen L., De Wannemacker G. et MOENS L., "Capabilities of inductively coupled plasma spectrometry for the measurement of Fe isotope ratios", $J$. Anal. At. Spectrom., 17, 2002, 933-943.

[24] Vanhoe H., Vandecasteele C., Versieck J. et DAMS R., "Determination of Iron, Cobalt, Copper, Zinc, Rubidium, Molybdenum, and Cesium in human serum by Inductively Coupled Plasma Mass Spectrometry", Anal. Chem., 61, 1989, 1851-1857.

[25] Moens L., Verrept P. et DAMS R., "New highresolution Inductively Coupled Plasma Mass Spectrometry technology applied for the determination of $\mathrm{V}, \mathrm{Fe}, \mathrm{Cu}, \mathrm{Zn}$ and $\mathrm{Ag}$ in human Serum”, J. Anal. At. Spectrom., 9, 1994, 1074-1078.

[26] Riondato J., Vanhaecke F., Moens L. et Dams R., "Determination of trace and ultra trace elements in human serum with a Double Focusing Magnetic Sector Inductively Coupled Plasma Mass Spectrometer", J. Anal. At. Spectrom., 12, 1997, 933-937.

[27] SARIEGo Muniz C., Marchante Gayón J.M., García Alonso J.I. et SANZ-MEDEL A., "Multi-elemental trace analysis of human serum by double-focusing ICP-MS", $J$. Anal. At. Spectrom., 14, 1998, 193-198.

Article reçu le 19 janvier 2015, version révisée reçue le 24 juillet 2015.
[28] Hinojosa Reyes L., MaRchante Gayón J.M., García ALONSO J.I. et SANZ-MEDEL A., "Determination of selenium in biological materials by isotope dilution analysis with an octapole reaction system ICP-MS", J. Anal. At. Spectrom., 18, 2003, 11-16.

[29] YU L.L., DAVIS W.C., ORdoneZ Y.N. et Long S.E., "Fast and accurate determination of $\mathrm{K}, \mathrm{Ca}$, and $\mathrm{Mg}$ in human serum by sector field ICP-MS", Anal. Bioanal. Chem., 405, 2013, 8761-8768.

[30] Simpson L.A., Hearn R., Merson S. et Catterick T., "A comparison of double-focusing sector field ICPMS, ICP-OES and octopole collision cell ICP-MS for the high-accuracy determination of calcium in human serum", Talanta, 65, 2005, 900-906.

[31] Compte-rendu de la $4^{\mathrm{e}}$ réunion du Comité Consultatif pour la Quantité de Matière, (1998), 1999, accessible par le site web du BIPM : http://www.bipm.org.

[32] «Annales du contrôle national de qualité des analyses de biologie médicale - Décembre 2012 », ANSM, 2014. http://ansm.sante.fr/var/ansm_site/storage/original/ application/de265b4ea27e79bc6b09c8a926e8b479.pdf

[33] Fassett J.D. et PAUlsen P.J., "Isotope dilution mass spectrometry for accurate elemental analysis", Anal. Chem., 61, 1989, 643-649.

[34] STUMPF C. et LABARRAQUE G., « La dilution isotopique par ICP/MS : une méthode de référence pour l'analyse d'éléments traces », Spectra Analyse, 32, 2003, 14-18.

[35] JCGM 100:2008 «Évaluation des données de mesure Guide pour l'expression de l'incertitude de mesure / Evaluation of measurement data - Guide to the expression of uncertainty in measurement », (GUM 1995 avec des corrections mineures), accessible par le site web du BIPM : http://www.bipm.org/fr/publications/guides/.

[36] NF EN ISO 13528 «Méthodes statistiques utilisées dans les essais d'aptitude par comparaisons interlaboratoires », AFNOR, 2005.

[37] Alimonti A., Bocca B., Mannella E., Petrucci F., Zennaro F., Cotichini R., D'ippolito C., AgResti A., CAIMI S., FORTE G., "Assessment of reference values for selected elements in a healthy urban population", Ann Ist Super Sanità, 41, 2005, 181-187.

[38] Nixon D.E., MOYeR T.P., Johnson P., Mccall J.T., Ness A.B., FJERStad W.H. et Wehde M.B., "Routine measurement of Calcium, Magnesium, Copper, Zinc, and Iron in urine and serum by Inductively Coupled Plasma Emission Spectroscopy", Clin. Chem., 32, 1986, 1660-1685. 